\title{
IMPLANTAÇ̃̃O DE BIM EM UMA CONSTRUTORA DE MÉDIO PORTE: CASO PRÁTICO, DA MODELAGEM A QUANTIFICAÇÃO
}

\section{BIM IMPLEMENTATION WTHINAMEDIUM-SIZED CONSTRUCTION FIRM: A CASE STUDY FROM MODELING TO QUANTTTY TAKE-OFF}

\author{
Romeu da Silva Neiva Neto \\ Brayer Luiz de Faria
}

Sérgio Adriano Bizello

\begin{abstract}
Information and Communication Technologies has been, in recent years, gaining ground in building construction. In Brazil, Building Information Modeling (BIM) adoption in construction firms around the country is an evidence of such growth. It first happened in firms near big economic centers; however now interest is spreading throughout the rest of the territory. This article aims to explain briefly the steps taken for BIM Implementation at a corporate building project in Aparecida de Goiânia. The BIM implementation process was done in collaboration with the University of Campinas in an action research. The research applied modeling practices and model management activities to evaluate the benefits of a model-based approach at a construction firm design process. The accomplished benefits are relate to error reduction and omissions in design and the precision of quantity takeoff in the estimate process.
\end{abstract}

Keywords: BIM. Implementation. Construction. Real State.

Romeu da Silva Neiva Neto

INNOVAR Construtora, Goiânia - GO, romeuneiva@gmail.com

Brayer Luiz de Faria

Universidade Estadual de Campinas (UNICAMP), Faculdade de Engenharia

Civil, Arquitetura e Urbanismo, Curso de Engenharia Civil, Campinas - SP brayerlf@gmail.com

Sérgio Adriano Bizello

Universidade Estadual de Campinas

(UNICAMP), Faculdade de Engenharia Civil, Arquitetura e Urbanismo, Coordenadoria de Projetos, Laboratório LaBPRAXIS, Campinas - SP, bizello@fec.unicamp.br

\section{Introdução}

A Modelagem da Informação da Construção, ou a sigla internacional mais conhecida como BIM, veio para ficar entre os processos do mercado de Arquitetura, Engenharia e Construção (AEC) de todo o mundo. Várias discussões vêm sendo feitas ao longo dos anos de como esta seria uma alternativa tecnológica para a melhoria dos processos na construção civil (EASTMAN et al., 2011). Atualmente tem-se total ciência que é um caminho sem volta a adoção dessas novas tecnologias aos processos das empresas. Cabe apenas aos pioneiros, dar o primeiro passo nessa direção e começar aos poucos absorver os investimentos e visualizar as vantagens de se ter novos processos, novas políticas e novas tecnologias para o mercado de AEC (SUCCAR, 2009).

Dentro desta nova realidade existem diversas barreiras que impedem inicialmente, para empresas da construção civil e seus intervenientes, um investimento mais incisivo com esse tipo de inovação (WON et al., 2013). O investimento inicial para se realizar uma implantação de BIM em uma construtora ou incorporadora ainda é muito alto e as barreiras no acesso à informação e ao processo de transformação requerido, também são fatores que contribuem para a resistência.

Entretanto, observa-se fatores nos últimos 10 anos que corroboram para a transposição destas barreiras. Um deles é o uso em escritórios de arquitetura de ferramentas de modelagem BIM para o projeto. Isso possibilitou uma vantagem competitiva de alguns escritórios de arquitetura que começaram a desenvolver seus projetos com maior produtividade e consistência (JUSTI, 2008; SOUZA; LYRIO, 2009). Da mesma forma, mas com maior impacto, as construtoras começaram a enxergar as

How to cite this article:

NEIVA NETO, Romeu da Silva; FARIA, Brayer Luiz de; BIZELLO, Sérgio Adriano. Implantação de BIM em uma construtora de médio porte: caso prático, da modelagem a quantificação. PARC Pesquisa em Arquitetura e Construção, Campinas, v. 5, n.1, p. 45-51, jan./jun. 2014. 
vantagens no uso da modelagem BIM nos empreendimentos para a compatibilização de projetos, o planejamento da obra e a orçamentação da mesma. Todas essas ações colaboraram para o pioneirismo de alguns empreendedores da construção nacional, começarem a investir na implantação de BIM. Isso vem se tornando uma realidade crescente entre as construtoras e incorporadoras brasileiras, conforme uma recente pesquisa feita pelo Instituto Internacional Mcgraw Hill Construction (McGRAW, 2014).

Este artigo descreve o caso de implantação de BIM de uma construtora brasileira localizada em Goiânia, Goiás. O caso exemplifica o vencimento das barreiras existentes para esse investimento no país e demonstra como essa situação pode ser vencida quando o escritório de arquitetura em busca de produtividade, investe no desenvolvimento dos projetos arquitetônicos em uma plataforma BIM e viabiliza a implantação na construtora, além de contar com a colaboração do embasamento teórico de uma Universidade no caso em questão.

\section{BIM para construtoras}

O processo de implantação de BIM em uma empresa da área da Arquitetura, Engenharia e Construção (AEC) pode variar de acordo com a estratégia da organização, usos pretendidos do BIM, processos existentes, informação compartilhada, infraestrutura operacional (equipamentos, aplicativos e espaço) e pessoas envolvidas (COMPUTER, 2012). Meireles (2013) e Gu e London (2010) apresentam orientações para o processo de implantação aplicáveis em diferentes áreas.

Construir uma edificação é uma operação complexa. Cada empresa construtora cria métodos e maneiras de executar os serviços necessários, tanto para si mesmo, como para verificar ou medir serviços terceirizados, procurando manter a qualidade e uniformidade do produto final. Além disso, cada empresa pode ter uma ou mais deficiências consideradas que pretende (e precisa) sanar, sendo que resolver tais problemas se tornam o objetivo primeiro da implantação de BIM. Neste contexto, o processo de implantação se torna quase único para cada caso. É possível também investigar experiências similares a fim de não "reinventar a roda", acelerando o processo (MEIRELES, 2013). De qualquer maneira, o entendimento por parte da gerência e demais membros da empresa, de o que é um modelo BIM e de como isso pode dar suporte aos processos de trabalho, é muito importante (EASTMAN et al., 2011)

O caso aqui apresentado desenvolveu-se como uma pesquisaação em ciclos para determinar: Objetivos iniciais, Modelagem 3D de projetos do empreendimento, Especificação do modelo 3D e Extração de quantitativos para orçamento. O processo foi interativo, pois entre cada ciclo do processo, bem como durante a execução do mesmo, ocorreram momentos de revisão dos processos.

\section{Objetivos inicias}

Para que uma implantação de BIM possa ser bem sucedida, independente da área de atuação da empresa do mercado de AEC que deseja realizar a implantação, é necessário descrever quais são os objetivos dela com BIM. Ou seja, quais os "usos" de BIM que a empresa pode adotar para melhorar os processos falhos que a empresa possui devido à um processo "tradicional” em CAD. O CIC Research Group (COMPUTER, 2012), descreve esses usos de BIM ao longo das etapas do ciclo de vida de uma edificação e demonstra como uma implantação de BIM pode atuar nas etapas de planejamento, projeto (design), construção e operação.

Para o caso realizado, as aplicações foram feitas essencialmente focando a etapa de Projetos do empreendimento em questão. Sendo assim, foi aplicada a coordenação 3D de projetos (Design 3D Coordination) visando melhorar a qualidade da construção por meio da identificação de interferências e da precisão da quantificação para a orçamentação.

\section{0 processo de desenvolvimento}

O empreendimento, do caso prático aqui relatado, consistiu em dois edifícios de 20 pavimentos cada para uso comercial na região de Aparecida de Goiânia em Goiás (Figura 1).

Figura 1. Empreendimento estudado

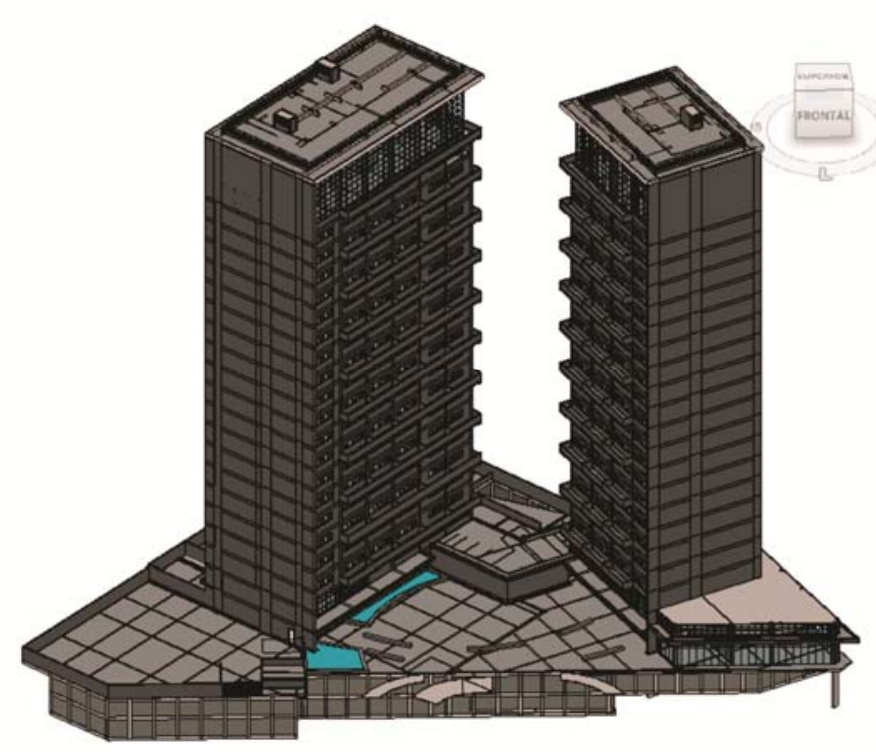

Fonte: Os autores

O caso prático envolveu o desenvolvimento de projeto das disciplinas de Arquitetura, Estruturas e Sistemas Prediais (Figura 2). O projeto de estruturas envolveu a superestrutura em concreto armando e a fundação. O projeto de sistemas prediais envolveu os projetos elétrico e de telefonia, hidro sanitário, de incêndio e de ar condicionado. Alguns projetos foram originalmente desenvolvidos em BIM na ferramenta de 
modelagem REVIT ou TQS e outros foram originalmente modelados em CAD e remodelados para BIM, conforme demonstrado na Figura 2. O processo de modelagem seguiu dois ciclos (Figura 3) enfatizando primeiramente os requisitos para o desenvolvimento do projeto executivo tendo como mote central a compatibilização 3D no NavisWorks. O segundo ciclo de modelagem enfatizou o processo de quantificação a partir do modelo BIM para a orçamentação.

Todas as disciplinas modeladas obedeceram à uma especificação de acordo com o objetivo de cada etapa no desenvolvimento dos projetos. Cada ciclo de modelagem além de ter como produto final um modelo BIM também resultava num conjunto de orientações para a modelagem.
As atividades de recebimento dos modelos, avaliação, criação de padrões foi realizada pela construtora INNOVAR em colaboração com o Laboratório de Práticas Projetuais (LabPRAXIS) vinculado a Coordenadoria de Projetos (CPROJ) da Faculdade de Engenharia Civil, Arquitetura e Urbanismo (FEC) da Universidade Estadual de Campinas (UNICAMP). Esta colaboração foi possível por meio de um em convênio de pesquisa entre o LabPRAXIS e a INNOVAR. A modelagem BIM ou a remodelagem dependendo da disciplina de projeto era desenvolvida pelos projetistas responsáveis ou a equipe de pesquisa. A colaboração entre projetistas, construtora (um Engenheiro Civil e um Estagiário da INNOVAR) e equipe de pesquisa (um Tecnólogo em Construção Civil do LabPRAXIS/CPROJ), ocorreu ao longo de 6 meses em 2013.

Figura 2. Composição do projeto, tipos de modelos associados e modelagens desenvolvidas

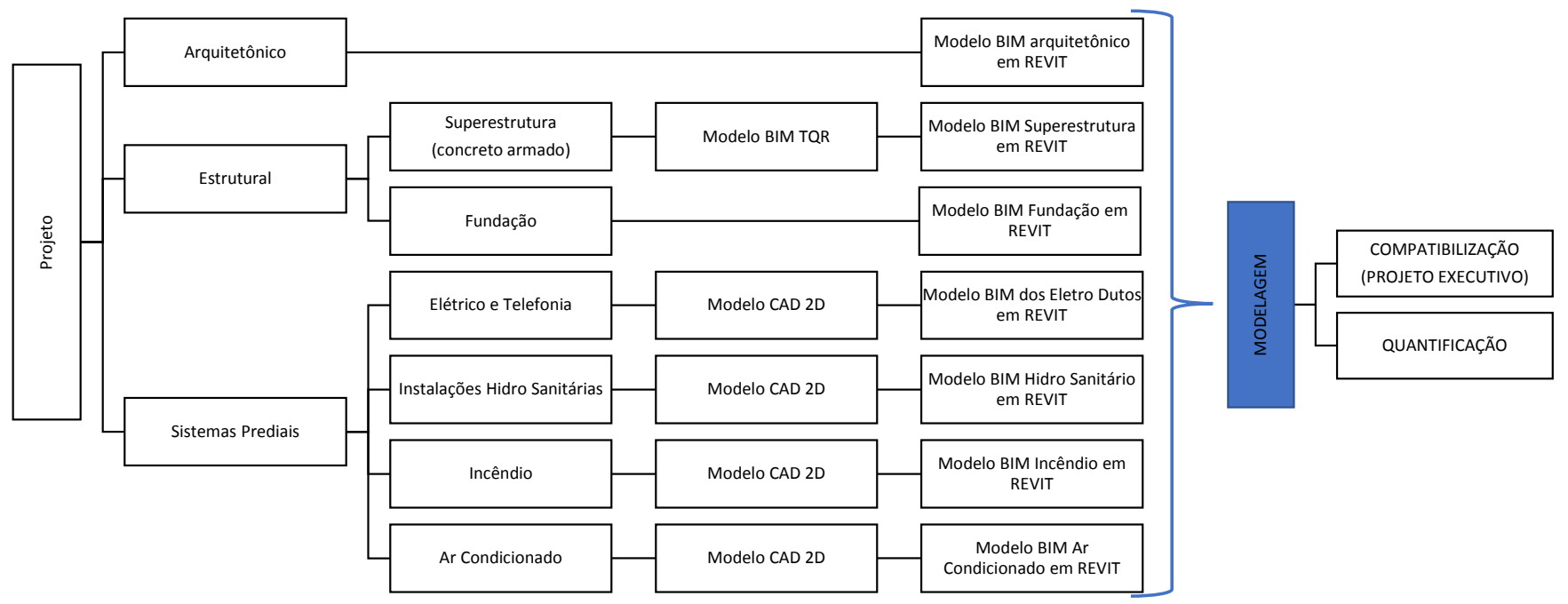

Fonte Os autores

Figura 3. Fluxo de modelagem

\section{1․ Ciclo ênfase nos requisitos de projeto executivo}

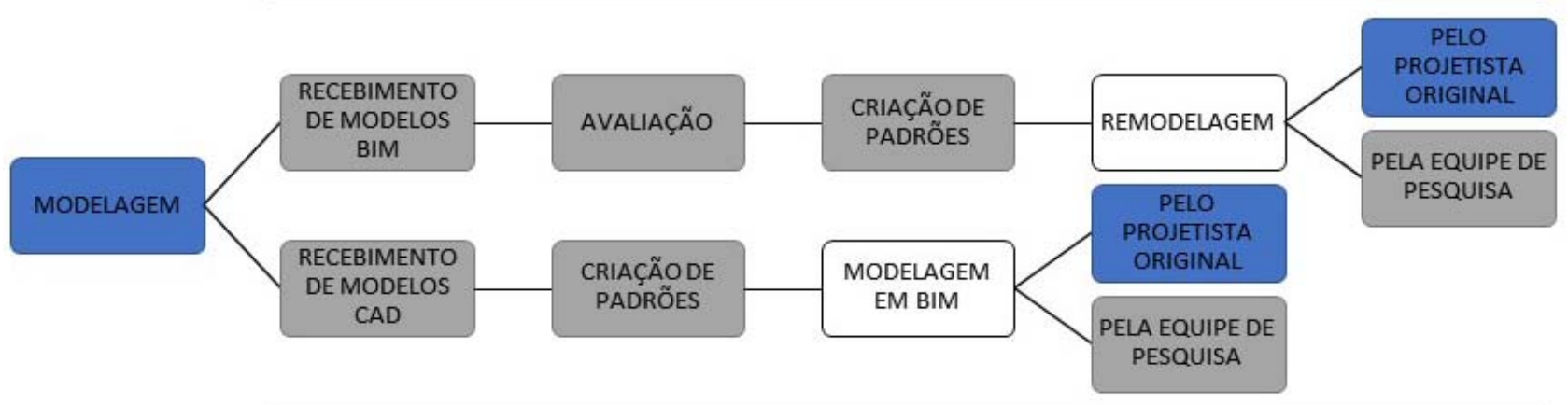

2․ Ciclo ênfase nos requisitos de quantificação

Fonte Os autores 


\section{Modelagem visando o Projeto Executivo}

Na primeira iteração incluindo recebimento de modelos, avaliação e criação de padrões observou-se as seguintes demandas:

- Separação dos modelos de Estrutura e Arquitetura em modelos referenciados para a melhoria na manipulação dos arquivos;

- Utilização de famílias (componentes) de instalações hidro sanitárias da biblioteca dos fabricantes apropriados;

- Revisão do pré-dimensionamento estrutural do modelo do Projeto da Estrutura de Concreto Armado devido a inconsistências na interoperabilidade entre as ferramentas de projeto.

As interferências encontradas entre os modelos de Arquitetura, Estrutura e Fundações foram feitas diretamente no momento da modelagem no REVIT e na verificação dos modelos dessas disciplinas através de checagens automatizadas ou feitas manualmente na mesma ferramenta.

Após essa análise de interferências entre os Projetos Arquitetônico e o Estrutural, ainda na ferramenta de modelagem, foi feito o clash detection entre a Arquitetura e os modelos dos Projetos de Sistemas Prediais (elétrico/telefônico, hidráulico, incêndio e ar-condicionado). Também foi feita a checagem entre a Estrutura e os modelos de sistemas prediais e pôr fim a checagem entre os próprios modelos de sistemas prediais (elétrico/telefônico com hidráulico, hidráulico com arcondicionado, e etc) (Figura 4). Ao final dessa análise, todos as informações de conflitos foram documentadas em forma de Relatórios com imagens 3D (Figura 5) e repassadas para os projetistas fazerem os ajustes dos projetos em 2D para o envio à obra.

O repasse das informações bem como a própria etapa de compatibilização pode ser feita de forma eletrônica, inclusive em tempo real, por meio de vídeo conferências o que permite a utilização de equipes geograficamente distantes.

\section{Modelagem visando a extração de quantitativos para orçamento}

A última etapa do desenvolvimento do trabalho consistiu no ajuste da modelagem para a extração de quantitativos para o orçamento da obra. Os modelos utilizados para esse fim foram os modelos dos Projetos Arquitetônicos e Estrutural. Os modelos dos Projetos de Sistemas prediais não foram utilizados para a quantificação, pois os projetistas que desenvolveram os pré-projetos de sistemas prediais em 2D, já forneciam quantitativos dos componentes as serem gastos na execução da obra.
Figura 4. Etapas da compatibilização

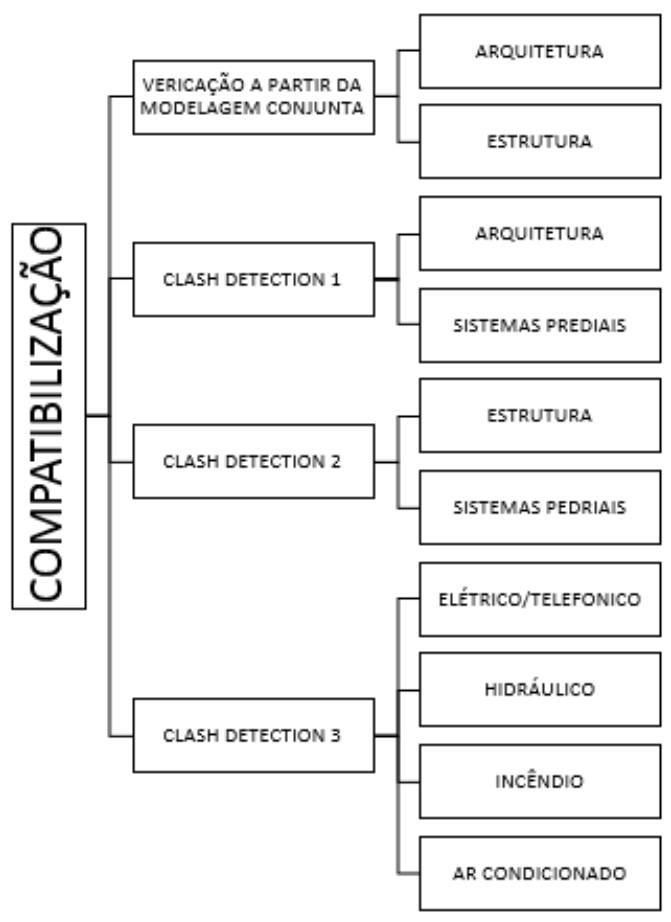

Fonte: Os autores

Figura 5. Imagens 3D para o relatório de interferências.

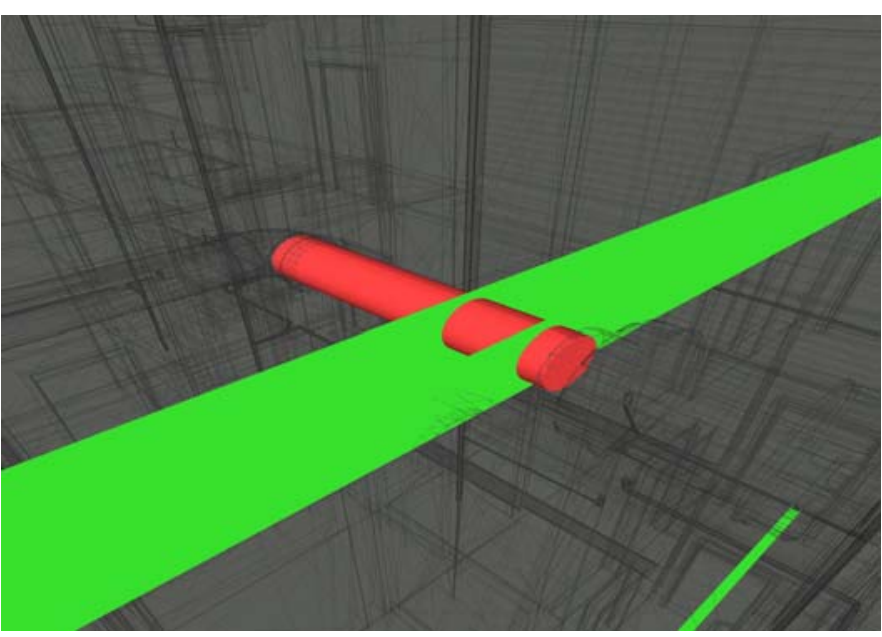

Fonte: Os autores

É preciso salientar que durante o processo de modelagem várias soluções foram experimentadas a fim de que se chegasse àquela que melhor representasse os processos da empresa, para os fins definidos. A Figura 6 exemplifica o nível de detalhamento adotado para representar a realidade a ser construída e o uso de BIM almejado: compatibilização e quantificação.

Os arquivos para a extração de quantitativos foram separados dos arquivos utilizados para a documentação, pois as alterações na modelagem iriam afetar a documentação do projeto. Sendo assim, a única documentação que fora extraída destes novos modelos, seriam as Tabelas de quantitativos para o orçamento. Porém, as alterações que estavam sendo feitas nos modelos 
executivos, eram relatadas em forma de relatórios de acompanhamento e replicadas aos modelos de quantitativos, possibilitando a troca de informação entre as duas atividades (Figura 7).

Figura 6. Padrão para componentes (famílias) de parede para a modelagem arquitetônica.

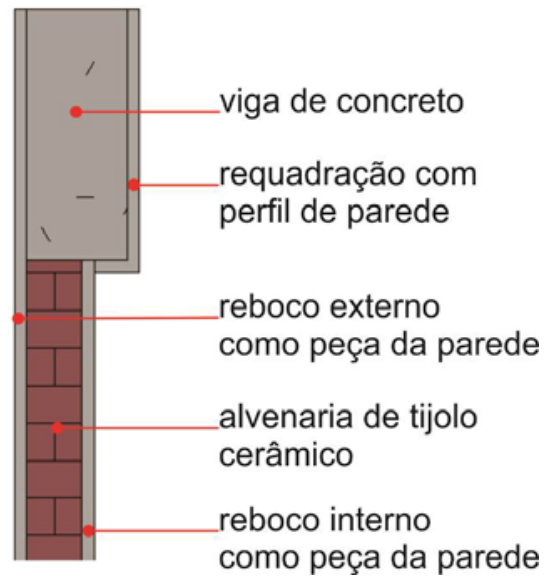

Fonte: Os autores

Figura 7. Esquema para demonstração da separação dos modelos executivos e os modelos para quantificação

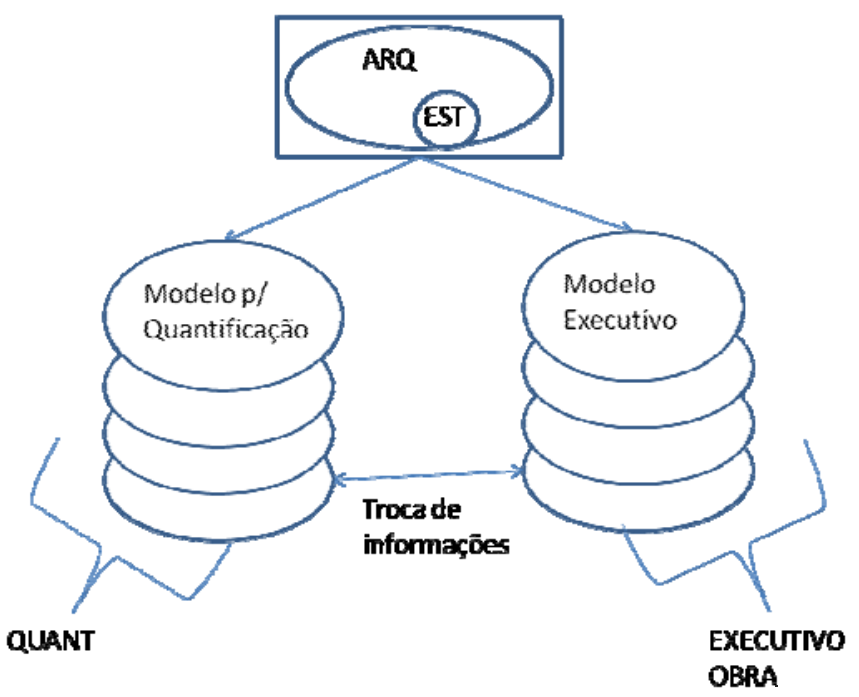

Fonte: Os autores

Pode-se considerar como ideal que todas as atividades, tanto a de produção documental para a obra (desenhos do projeto executivo) quanto a de extração das quantidades e suas respectivas planilhas sejam oriundas dos mesmos modelos arquitetônico, estrutural e de especialidades. Entretanto, devese levar em conta outros aspectos como, por exemplo, o tamanho dos arquivos, procurando minimizar os problemas durante sua manipulação.

Para o ajuste da modelagem para a quantificação, várias medidas foram tomadas de acordo com cada ítem do orçamento da obra que necessitava de quantitativos para a precificação.
Figura 8. Respectivamente, parede original e dividida, utilizando a funcionalidade "parts" ou peças do REVIT, permitindo a alteração e quantificação específicas

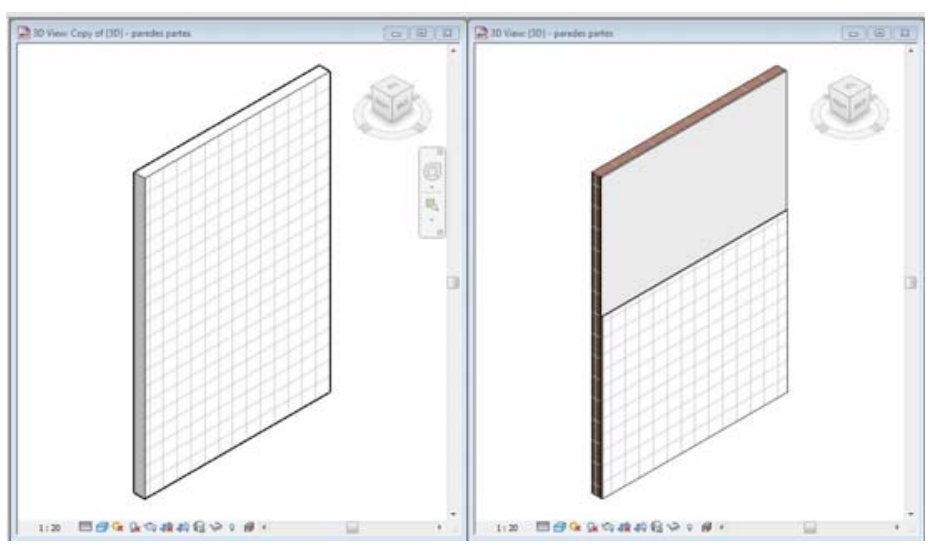

Fonte: Os autores.

$\mathrm{Na}$ parte de quantificação de alvenarias, revestimentos e pinturas de parede, piso e teto, foi adotado o uso das "parts" ou peças das famílias (componentes) do modelo arquitetônico. Essa funcionalidade do REVIT possibilitou a separação das camadas de alvenaria e revestimento das paredes, por exemplo (Figura 6 e 8). Dessa forma o modelo arquitetônico poderia ser quantificado de forma precisa, e esses quantitativos espelhariam os serviços a serem executados na obra. Esses serviços, por sua vez estariam itemizados na planilha de orçamento do sistema de gerenciamento da construtora, associadas (de forma manual) aos modelos tridimensionais do empreendimento.

É preciso saber que de acordo com a ferramenta BIM (software) utilizada, uma solução pode ser mais adequada ou não, pois a funcionalidade pode não existir. Desta mesma forma uma ferramenta pode ter quantitativos extraídos diretamente ou com mais facilidade do que outra.

\section{Conclusão}

As etapas de uma implantação de BIM em uma construtora dependem de quais são os objetivos que a mesma quer alcançar com o uso de BIM em seus processos. Sendo assim, a pesquisa-ação desenvolvido na construtora do caso em questão focou na análise de interferências entre os projetos para a redução dos erros e omissões de projeto e na quantificação dos serviços para o orçamento da obra. O que possibilitou uma melhor rastreabilidade da informação para a estimativa do custo da obra.

Esses objetivos adotados possibilitaram uma melhoria no gerenciamento de projetos da construtora que até o início da Implantação de BIM, dependia da análise manual e ineficiente das interferências de projeto. Essa análise era ineficiente tanto pela parte do projetista arquitetônico quanto pela parte do departamento responsável pelo gerenciamento das informações na construtora, o que incorria em inúmeros erros e omissões de projetos, causando atrasos e desperdícios em obra. Sendo assim, ao final da Implantação, identificou-se a vantagem de 
Quadro 1. Tabela de identificação das etapas a serem quantificadas por partes e a sua forma de visualização no modelo arquitetônico

\begin{tabular}{|c|c|c|c|c|}
\hline & \multicolumn{2}{|c|}{ MODELO } & \multicolumn{2}{c|}{ MODELO DE VISUALIZAÇÃO } \\
\hline & $\begin{array}{c}\text { MODELOS PARA } \\
\text { QUANTIFICAÇÃO }\end{array}$ & MODELOS EXECUTIVOS & VISUALIZAÇÂO ORIGINAL & $\begin{array}{c}\text { VISUALIZAÇÂO DE } \\
\text { PEÇAS }\end{array}$ \\
\hline ALVENARIA & $\mathrm{X}$ & $\mathrm{X}$ & $\mathrm{X}$ & \\
\hline ESQUADRIA & & $\mathrm{X}$ & $\mathrm{X}$ & \\
\hline REVESTIMENTO INTERNO & & $\mathrm{X}$ & $\mathrm{X}$ \\
\hline REVESTIMENTO EXTERNO & $\mathrm{X}$ & $\mathrm{X}$ & $\mathrm{X}$ \\
\hline PINTURA & $\mathrm{X}$ & $\mathrm{X}$ & \\
\hline FORRO & & $\mathrm{X}$ & \\
\hline PISO & & $\mathrm{X}$ & \\
\hline
\end{tabular}

Fonte: Os autores

se desenvolver os projetos com a tecnologia de modelagem BIM, pois grande parte desses erros e omissões foram identificados na etapa de projeto. Portanto, o recebimento de projetos em BIM dentro dos padrões estabelecidos nesta pesquisa passou a ser uma premissa para a contratação de futuros projetos da empresa. A separação de modelo de informação para quantificação e modelo de informação para projetos executivos também foi adotada.

Quando se trata da orçamentação, a análise feita deve acompanhar o processo de execução da obra. A validação é feita através de todos os dados levantados ao início da obra comparados aos dados levantados ao longo da construção e ao final da mesma. Como a obra encontra-se em andamento, pretende-se apresentar esses resultados em futuras publicações a respeito do projeto de pesquisa.

Existem diversas outras formas de se abordar os problemas ocorridos nos processos de construtoras e incorporadoras, porém o valor adquirido com essas iniciativas é o passo inicial para o amadurecimento dos processos empresariais que cada organização irá trilhar. As lições aprendidas com esses exemplos serão o fundamento principal para a consolidação tanto do ensino de BIM nas universidades brasileiras, quanto da evolução dos conceitos de inovação presentes no mercado da Construção Civil brasileira.

\section{Notas}

(1) Interoperabilidade: é a capacidade de um sistema (informatizado ou não) de se comunicar de forma transparente (ou o mais próximo disso) com outro sistema (semelhante ou não). Para um sistema ser considerado interoperável, é muito importante que ele trabalhe com padrões abertos ou ontologias. Em um contexto BIM de projeto, o padrão mais conhecido é o Industry Foundation Classes (IFC).

\section{Referências}

COMPUTER Integrated Construction Research Program. BIM Project Execution Planning Guide. Version 2.0, The Pennsylvania State University, University Park, PA, USA, abr. 2012. Disponível em: <http://bim.psu.edu/default.aspx>. Acesso em: 17 jul. 2014.

McGRAW Hill Construction. The Business Value of BIM for Construction in Major Global Markets: How contractors around the world are driving innovations with Building Information Modelling. Smart MarketReport, 2014. Disponível em: < http://www.icn-solutions.nl/pdf/bim_construction.pdf>. Acesso em: 17 jul. 2014.

EASTMAN, C.; TEICHOLZ, P.; SACKS, R.; LISTON, K. BIM Handbook: A guide to Building Information Modeling for owners, managers, designers, engineers, and contractors. 2. ed. Hoboken, New Jersey: John Wiley \& Sons, 2011.626 p.

MEIRELES, A. R. Say “NO!” to Hollywood BIM: An advanced integration of the BIM process at a builder. AECbytes, Building the Future Article, set. 2013. Disponível em:

<http://www.aecbytes.com/buildingthefuture/2013/BIM_MotaEngil.html>. Acesso em: 17 jul. 2014.

JUSTI, Alexander Rodrigues. Implantação da plataforma REVIT nos escritórios brasileiros: relato de uma experiência. Gestão \& Tecnologia de Projetos, v. 3, p. 140-152, mai. 2008. Disponível em: <

http://www.revistas.usp.br/gestaodeprojetos/article/view/50931/55013>. Acesso em: 17 jul. 2014. 
SOUZA, Livia L. Alves de; AMORIM, Sérgio R. Leusin; LYRIO, Arnaldo de Magalhães. Impactos do uso do BIM em escritórios de arquitetura: oportunidades no mercado imobiliário. Gestão \& Tecnologia de Projetos, v. 4, p. 26-53, nov. 2009. Disponível em: <http://www.revistas.usp.br/gestaodeprojetos/article/view/50958>. Acesso em: 17 jul. 2014.

SUCCAR, Bilal. Building information modelling framework: A research and delivery foundation for industry stakeholders. Automation in Construction, [s.l.], v. 18, n. 3, p. 357-375, mai. 2009.

WON, J.; LEE, G.; DOSSICK, C.; MESSNER, J.. Where to Focus for Successful Adoption of Building Information Modeling within Organization. Journal of Construction Engineering and Management, v. 139, n. 11, nov. 2013. Disponível em: <http://ascelibrary.org/doi/full/10.1061/\%28ASCE\%29CO.1943-7862.0000731>. Acesso em: 17 jul. 2014. 